\title{
Thrifty metabolism that favors fat storage after caloric restriction: a role for skeletal muscle phosphatidylinositol-3-kinase activity and AMP-activated protein kinase
}

\author{
S. Summermatter, ${ }^{*}$ D. Mainieri, ${ }^{*}$ A. P. Russell, ${ }^{\ddagger}$ J. Seydoux,${ }^{\S}$ J. P. Montani, ${ }^{*}$ \\ A. Buchala, ${ }^{\dagger}$ G. Solinas, ${ }^{*}$ and A. G. Dulloo*,1 \\ *Department of Medicine, Division of Physiology, and ${ }^{\dagger}$ Department of Biology, University of \\ Fribourg, Fribourg, Switzerland; ${ }^{\ddagger}$ Centre for Physical Activity and Nutrition, School of Exercise \\ and Nutrition Sciences, Deakin University, Melbourne, Australia; and ${ }^{\mathrm{S}}$ Department of Basic \\ Neurosciences, Faculty of Medicine, University of Geneva, Geneva, Switzerland
}

\begin{abstract}
Energy conservation directed at accelerating body fat recovery (or catch-up fat) contributes to obesity relapse after slimming and to excess fat gain during catch-up growth after malnutrition. To investigate the mechanisms underlying such thrifty metabolism for catch-up fat, we tested whether during refeeding after caloric restriction rats exhibiting catch-up fat driven by suppressed thermogenesis have diminished skeletal muscle phosphatidylinositol-3-kinase (PI3K) activity or AMP-activated protein kinase (AMPK) signaling - two pathways required for hormone-induced thermogenesis in ex vivo muscle preparations. The results show that during isocaloric refeeding with a low-fat diet, at time points when body fat, circulating free fatty acids, and intramyocellular lipids in refed animals do not exceed those of controls, muscle insulin receptor substrate 1-associated PI3K activity (basal and in vivo insulin-stimulated) is lower than that in controls. Isocaloric refeeding with a high-fat diet, which exacerbates the suppression of thermogenesis, results in further reductions in muscle PI3K activity and in impaired AMPK phosphorylation (basal and in vivo leptin-stimulated). It is proposed that reduced skeletal muscle PI3K/AMPK signaling and suppressed thermogenesis are interdependent. Defective PI3K or AMPK signaling will reduce the rate of substrate cycling between de novo lipogenesis and lipid oxidation, leading to suppressed thermogenesis, which accelerates body fat recovery and furthermore sensitizes skeletal muscle to dietary fatinduced impairments in PI3K/AMPK signaling
\end{abstract}

Key Words: thermogenesis $\cdot$ weight cycling catch-up growth - obesity $\cdot$ diabetes
THE RECOVERY OF BODY WEIGHT after caloric restriction in humans and other mammals is generally accompanied by a disproportionately faster rate in recovering fat mass than lean body mass (1). This phenomenon of preferential "catch-up fat" is in part the consequence of an enhanced metabolic efficiency for fat deposition that occurs in response to fat depletion (2-9). It is viewed as the outcome of an "adipose-specific" control of thermogenesis (10)—an autoregulatory feedback system in which signals from the depleted adipose tissue fat stores exert a suppressive effect on thermogenesis until the fat stores are replenished, with the energy conserved during weight recovery being directed specifically to recovering fat mass. This thrifty metabolism for catch-up fat probably evolved to optimize survival capacity in an ancestral lifestyle characterized by periodic famine. However, in today's environment of food abundance, it contributes to the relapse of obesity after slimming and hence to the poor efficacy of dietary restriction in the management of obesity. Furthermore, this thrifty metabolism promotes preferential catch-up fat associated with insulin resistance during catch-up growth after earlier periods of faltered growth $(11,12)$ and has been implicated in the link between earlier growth retardation, catch-up growth, and increased risks for type 2 diabetes and cardiovascular disease later in life (13).

The molecular mechanisms underlying this adiposespecific control of thermogenesis are unknown. However, the demonstration that the preferential catch-up fat caused by suppressed thermogenesis persists unabated under both conditions of cold exposure (when sympathetically mediated thermogenesis is markedly elevated) and at thermoneutrality (when sympathetically mediated thermogenesis is minimal) (14) has

\footnotetext{
${ }^{1}$ Correspondence: Department of Medicine, Division of Physiology, University of Fribourg, Rue du Musée 5, CH-1700 Fribourg, Switzerland. E-mail: abdul.dulloo@unifr.ch
} 
indicated that this thrifty metabolism favoring catch-up fat can be dissociated from diminished sympathetic neural control of thermogenesis and, by extension, from sympathetic modulation of the uncoupling protein (UCP) 1 in brown adipose tissue. Subsequent interest in the possibility that such regulated thermogenesis in skeletal muscle may involve altered mitochondrial uncoupling by UCP2 or UCP3 (members of the UCP family) has waned after the demonstration that they are either up-regulated during fasting (15-18) or unaltered in response to semistarvation and refeeding (19). Indeed, a role for UCP2 and UCP3 in the control of thermogenesis and energy balance is still being debated (18-21).

There is now renewed interest in the potential role of futile (energy-dissipating) cycles in understanding the biochemical basis of skeletal muscle thermogenesis. In this context, mice with genetic ablation of stearoyl-CoA desaturase 1 (SCD1), a rate-limiting enzyme that catalyzes fatty acid desaturation at the $\Delta 9$ position, display a hypermetabolic phenotype $(22,23)$, which was proposed to be mediated by a futile cycle between de novo lipogenesis and lipid oxidation (24). Furthermore, the findings that after caloric restriction, rats refed with a chow diet showed elevations in SCD1 gene expression and in indices of SCD1-related fatty acid $\Delta 9$ desaturation in skeletal muscle raised the possibility for a role of increased muscle SCD1 activity in the suppressed thermogenesis driving catch-up fat (24). As the latter study was in progress, it was reported that the hypermetabolic SCD1-knockout mice display increased activation of insulin receptor substrate (IRS) 1-associated phosphatidylinositol-3-kinase (PI3K) as well as AMP-activated protein kinase (AMPK) in skeletal muscle $(25,26)$. We found these observations to be of particular interest in light of our ex vivo microcalorimetric studies in intact skeletal muscle demonstrating that the direct effects of leptin and corticotropin-releasing hormone in stimulating muscle thermogenesis correlate with AMPK and PI3K signaling and can be blocked by selective inhibitors of either AMPK or PI3K (27-29). These studies therefore strongly suggest that both PI3K and AMPK signaling are required for hormonal control of thermogenesis in skeletal muscle.

On the basis of these findings, we investigated here whether IRS1-associated PI3K activity or AMPK phosphorylation is impaired in skeletal muscle of rats during catch-up fat and could thus be implicated in the thrifty metabolism of suppressed thermogenesis that drives catch-up fat after caloric restriction. To this end, we have used a previously described rat model of semistarvation-refeeding, in which catch-up fat results solely from suppressed thermogenesis, to assess IRS1-associated PI3K activity and AMPK phosphorylation in two skeletal muscle types, tibialis anterior (a fast-twitch glycolytic muscle) and soleus muscle (a slow-twitch oxidative muscle), which were harvested under the unstimulated (basal) state as well as under in vivo stimulated states after administration of either insulin (an inducer of PI3K) or leptin (an activator of AMPK).
These investigations were first carried out during catch-up fat with a chow (low-fat) diet and subsequently were repeated during isocaloric refeeding with a highfat (typical Western) diet, which is known to further exacerbate the thrifty metabolism that drives catch-up fat (11). These studies are interpreted with data from concomitant assays of muscle lipid content, SCD1 gene expression, and SCD1-associated fatty acid $\Delta 9$ desaturation, as well as with data from blood or urinary assays of hormones implicated in the control of muscle thermogenesis (thyroid hormones, leptin, and catecholamines).

\section{MATERIALS AND METHODS}

\section{General study design}

Male Sprague-Dawley rats (Elevage Janvier, Le Genest Saint Isle, France), caged singly in a temperature-controlled room $\left(22 \pm 1^{\circ} \mathrm{C}\right)$ with a 12 -h light/dark cycle, were maintained with a commercial chow diet (Kliba, Cossonay, Switzerland) consisting (by energy) of $24 \%$ protein, $66 \%$ carbohydrates, and $10 \%$ fat and had free access to tap water. Animals were maintained in accordance with our institute's regulations and guide for the care and use of laboratory animals. The experiments were conducted after $1 \mathrm{wk}$ of adaptation to housing conditions. They were performed using experimental designs that were similar to those previously described in establishing a rat model for studying changes in energy expenditure that occur for accelerating fat deposition during refeeding after $2 \mathrm{wk}$ of caloric restriction, i.e., an approach that allows suppressed thermogenesis specific for fat recovery to be studied in the absence of confounding variables, such as body size, food intake, and differential rates of protein gain, on energy expenditure $(9,10)$.

\section{Design of study I: catch-up fat with chow diet}

In a first set of experiments (study 1), the specific design of which has been reported before $(9-11)$ and is depicted in Fig. $1 A)$, groups of 7-wk-old rats are food-restricted at $50 \%$ of their spontaneous chow intake for $2 \mathrm{wk}$. After this period of semistarvation, they are refed the same standard chow diet, which is low in fat and high in unrefined carbohydrates (Supplemental Table 1) at a level equal in metabolizable energy content to the spontaneous food intake of control rats matched for weight at the onset of refeeding. The cardinal feature of this experimental design, therefore, is that comparisons are made between refed animals regaining weight $v$. spontaneously growing weight-matched controls, with both groups consuming the same amount of food energy. Under these conditions, the refed animals show an increased rate of body fat gain for a period of 2-3 wk, owing to 10-13\% lower energy expenditure than controls (9-11) and confirmed here in Fig. 1A. A number of factors that could theoretically contribute to this difference in energetics between refed and control rats (namely, protein mass, size of organs, age, and level of physical activity) have been evaluated previously and were shown to have little or no impact on the difference in energy expenditure between the two groups $(9,10)$. The lack of between-group differences in lean body mass is also confirmed here as judged from our data presented in Supplemental Fig. 1 $A$, indicating that the body protein mass is similar in refed and control groups during the course of catch-up fat; hence, no correction is required for adjusting 
A
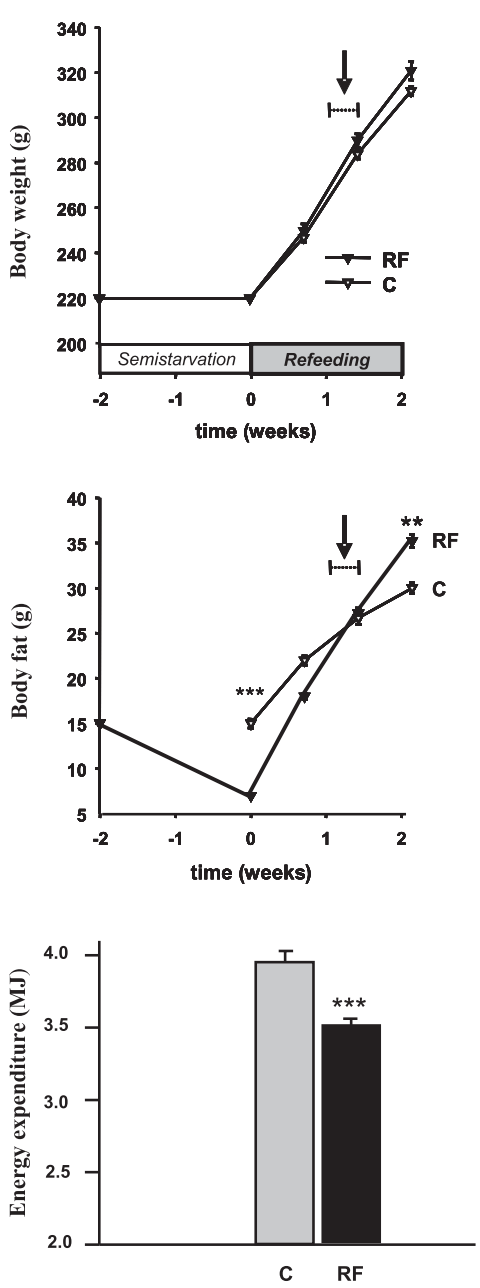

B
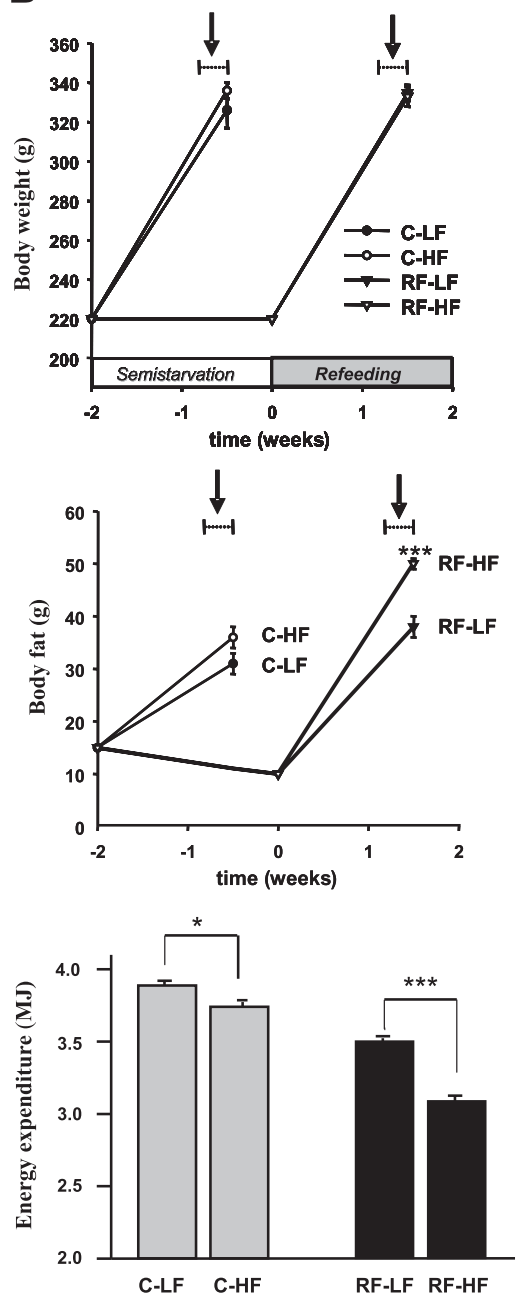

Figure 1. Experimental designs using a previously described rat model of semistarvationrefeeding in which the accelerated fat recovery (catch-up fat) results only from suppressed thermogenesis, i.e., not from the confounding effects of hyperphagia or lower lean body mass or protein mass $(9,10)$. In the investigations reported here, body composition measurements were determined at days $0,5,10$, and 15 of refeeding with a chow diet in refed (RF) and control $(\mathrm{C})$ animals in study design I $(A)$, and at days 0 and 10 of refeeding with high-fat (HF) or low-fat (LF) semisynthetic diets in refed and fed controls in study design II $(B)$. In study design II $(B), \mathrm{C}-\mathrm{LF}$ and C-HF are control groups fed on isocaloric amounts of the low-fat or high-fat diet, respectively; RF-LF and RF-HF are refed groups consuming isocaloric amounts of the low-fat or high-fat diet, respectively. The arrows indicate the time-points at which skeletal muscle was harvested. In parallel experiments, energy expenditure was also determined over a 2-wk period by a comparative carcass technique, i.e., from the difference between the gain in body energy and metabolizable energy intake (9). All values are means $\pm \mathrm{SE}(n=6) .{ }^{*} P<0.05 ; * * P<$ $0.01 ; * * * P<0.001$. energy expenditure relative to lean body mass for betweengroup comparison. Consequently, under conditions of our refeeding studies, the lower energy expenditure in refed than in control rats is explained essentially by the energy spared as a result of sustained suppression of thermogenesis for the purpose of catch-up fat. In the study reported here, the experiments were conducted for a period corresponding to 9 days of refeeding, i.e., the time point at which body fat of the refed animals had not yet exceeded that of controls (Fig. 1). In two separate experiments here, groups of refed and control animals $(n=12-18)$ were sacrificed after in vivo bolus administration of either insulin or saline (the PI3K experiment) and after in vivo bolus administration of leptin or saline (the AMPK experiment).

\section{Design of study II: catch-up fat with high-fat diet}

In a second set of experiments comparing semisynthetic diets high or low in dietary fats, the specific design of which is similar to that reported previously (11) and depicted in Fig. $1 B)$, groups of spontaneously growing rats, referred to as fed controls, were fed either the low-fat diet $(n=12)$ or an isocaloric amount of the high-fat diet rich in lard $(n=12)$ for a period of 10 days; the compositions of these semisynthetic diets are presented in Supplemental Table 1, and details of measurements of the metabolizable energy content of these diets have been reported previously (11). Two additional groups of rats were semistarved for 14 days as described above and during subsequent refeeding lasting 10 days, one group $(n=12)$ was refed with the low-fat diet and the other group $(n=12)$ was refed with isocaloric amounts of the high-fat diet. All groups were thus provided with the same amount of metabolizable energy intake, which corresponds to that consumed during spontaneous food intake of pelletted chow. Under these conditions, the suppression of thermogenesis that favors catch-up fat with the low-fat diet was found to be exacerbated during refeeding with the high-fat diet (11), findings that are confirmed here in Fig. $1 B$ as indicated by a more pronounced effect of the high-fat diet on energy expenditure in refed animals than in fed controls, namely, refed high-fat diet $<$ refed low-fat diet by $\sim 12 \% \quad(P<0.001)$ compared with control high-fat diet $<$ control low-fat diet by $4 \%(P<0.05)$. This exacerbated reduction in absolute energy expenditure is independent of differences in lean body mass, as judged from our data (shown in Supplemental Fig. 1B), indicating that there were no differences in body protein mass in refed (or control) groups fed with the high-fat and low-fat diets. In separate experiments of similar design, subgroups of refed and fed control animals were sacrificed on day 10 after in vivo bolus administration of either saline or insulin (PI3K experiments) and after in vivo bolus administration of saline or leptin (AMPK experiments).

\section{In vivo bolus administration of insulin and leptin}

On the last day of each experiment, the rats were fasted from 7:00 AM, and 5-7 h later (i.e., between 12:00 and 2:00 PM), 
subgroups of rats were anesthetized by injection of ketamine/ xylazine (39:5 mg/ kg body weight) and surgically prepared for a bolus injection (through the jugular vein) of either insulin (10 U/kg body weight) (Actrapid, Novo Nordisk, Bagsvaerd, Denmark), recombinant leptin (1 $\mathrm{mg} / \mathrm{kg}$ body weight) (Insight Biotechnology, Wembley, UK), or an equal volume of saline vehicle. In the AMPK experiments, leptin or saline was injected 15 min before sacrifice of animals and tissue harvest, whereas in the PI3K experiments, insulin or saline was injected 3 min before sacrifice and tissue harvest. Immediately after sacrifice, the skeletal muscles (tibialis anterior and soleus) were rapidly dissected, frozen in liquid nitrogen, and stored at $-80^{\circ} \mathrm{C}$ until analysis. The timing for tissue harvesting after hormonal administration and the doses of hormones used correspond to maximal activation of PI3K and AMPK assessed in preliminary studies, consistent with values obtained by others $(30,31)$.

\section{Protein extraction and Western blotting}

Frozen tissues were homogenized under liquid nitrogen and incubated in lysis buffer [ $20 \mathrm{mM}$ Tris-HCl, $138 \mathrm{mM} \mathrm{NaCl}, 2.7$ $\mathrm{mM} \mathrm{KCl}, 5 \%$ (v/v) glycerol, $1 \%(\mathrm{v} / \mathrm{v})$ Nonidet P-40, and various hydrolase inhibitors] for $15 \mathrm{~min}$. After centrifugation at $15,000 \mathrm{rpm}$ for $15 \mathrm{~min}$, the protein concentration in the supernatant was quantified, and the protein extracts were used for further measurements. For Western blot analysis of phosphorylation of AMPK (Thr-172) and its downstream target acetyl-CoA carboxylase (ACC) (Ser-79, equal amounts of proteins were separated by SDS-PAGE and blotted on polyvinylidene difluoride membranes that were analyzed with polyclonal antibodies (Cell Signaling Technology, Inc., Danvers, MA, USA) raised against the indicated phosphorylation sites. Bands within the linear range were quantified densitometrically. Total protein amounts of AMPK $\alpha$ and ACC were also measured in the same way by using corresponding antibodies.

\section{PI3K activity}

Protein extract (200-500 mg) was immunoprecipitated with IRS-1 (Upstate Biotechnology, Lake Placid, NY, USA) polyclonal antibody. The kinase reaction, thin-layer chromatography separation, and signal detection were performed as described previously $(28,29)$ and according to the method of Pirola et al. (32).

\section{Intramyocellular lipids and SCD1-related fatty acid $\Delta 9$ desaturation}

The content of neutral lipids in skeletal muscle myocytes was determined using an oil red $\mathrm{O}$ stain, as described previously (12) and according to the method of Russell et al. (33). An index of SCD1-related fatty acid $\Delta 9$ desaturation was determined using the quantitated values for palmitate (C16:0), palmitoleate (C16:1), stearate (C18:0), and oleate (C18:1) in the phospholipid fraction as well as in the triglyceride fraction of muscle lipids obtained after fractionation by thin-layer chromatography and subsequent analysis of fatty acid methyl esters by gas chromatography/mass spectrometry, as detailed previously (24). The $\Delta 9$ desaturation index was calculated as the ratio of product to substrate (C16:1/C16:0 and C18:1/ C18:0).

\section{SCD1 expression}

The gene expression analysis for SCD1 was performed by real-time polymerase chain reaction, as detailed previously
(24), using iQ SYBR Green Supermix (Bio-Rad Laboratories, Hercules, CA), with the following primers: cyclophilin forward 5' TCA GGG CTC TTG AAG TCC C 3' and reverse 5' CAG AAA ATC ACA GCA GCC AAC 3'; and rat SCD1 forward $5^{\prime}$ TGG GAA AGT GAA GCG AGC AAC CG 3' and reverse 5' AGA GGG GCA CCT TCT TCA TCT TCT C 3'.

\section{Plasma hormones and urinary catecholamines}

Plasma thyroid hormone (T3) and leptin were measured using RIA kits (Linco Research, St. Charles, MO, USA). Catecholamines were assayed from urine collected during the last 4 days of the refeeding period using liquid chromatography with electrochemical detection, as described previously (24).

\section{Body composition}

The whole carcasses were dried to a constant weight in an oven maintained at $70^{\circ} \mathrm{C}$ and subsequently homogenized for analysis of energy content by bomb calorimetry (34) and for fat content by the Soxhlet extraction method (35). Body protein was determined from a general formula relating energy derived from fat, the total energy value of the carcass, and energy derived from protein (9).

\section{Data analysis and statistics}

All data are presented as means \pm SE. For the study comparing refed and control animals in response to hormone or saline, the data were analyzed by two-factor ANOVA for the main effects of groups (refed vs. control), treatment (hormone $v s$. saline), and group $\times$ treatment interactions. For the other study comparing animals consuming the high-fat and low-fat diets in response to hormone or saline, the data were analyzed by two-factor ANOVA for the main effects of diet (high-fat vs. low fat), treatment (hormone vs. saline), and diet $X$ treatment interactions; these analyses were conducted separately under conditions of feeding or refeeding. For both studies, post hoc pairwise comparisons were performed by unpaired $t$ test. The statistical treatment of data was performed using the computer software STATISTIK (version 4.0; Analytical Software, St. Paul, MN, USA).

\section{RESULTS}

\section{Study I: catch-up fat with chow diet}

\section{PI3K activity}

The data on IRS-1 associated PI3K activity in skeletal muscles from refed and control animals after saline administration (basal PI3K activity) or after in vivo insulin administration (insulin-stimulated PI3K activity) are presented in Fig. 2. In both tibialis anterior and soleus muscles, PI3K activity was found to be lower in refed than in control animals, with ANOVA indicating a statistically significant overall group effect (refed vs. control: $P<0.001$ ). These between-group differences are observed for basal PI3K activity, which is lower in both skeletal muscles of refed animals than in those of controls, namely by $22 \%(P<0.01)$ in tibialis anterior and by $19 \%(P<0.05)$ in soleus. Furthermore, insulinstimulated PI3K activity was also found to be lower in 

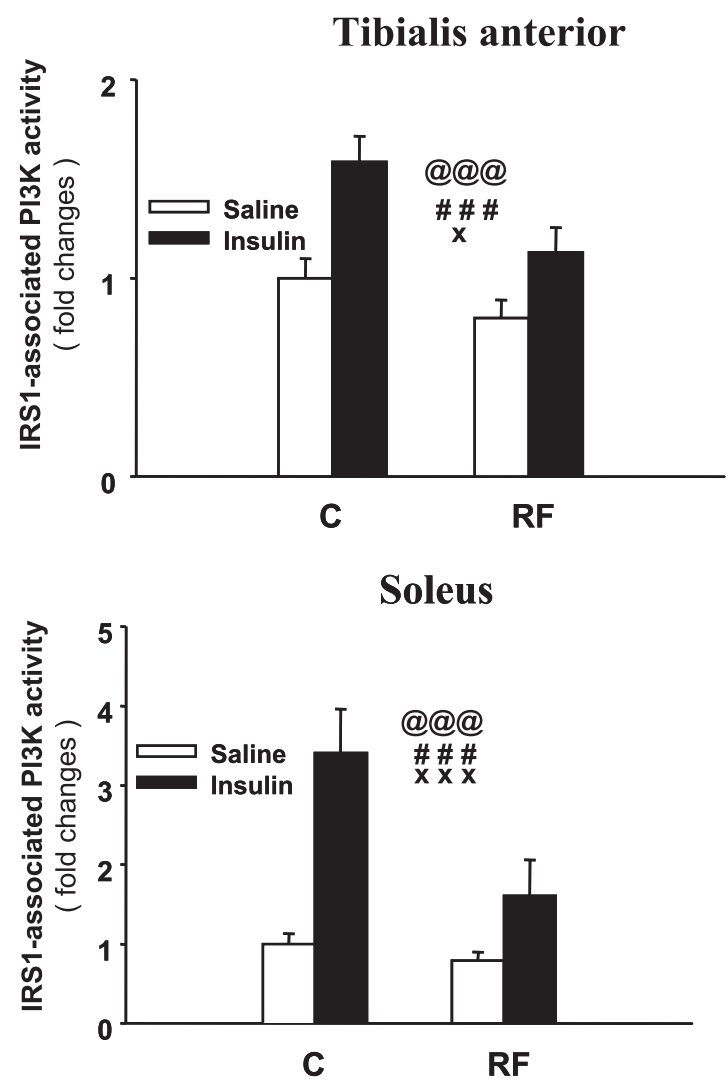

Figure 2. IRS1-associated PI3K activity in tibialis anterior and soleus muscles in response to either an acute bolus intravenous administration of $10 \mathrm{U} / \mathrm{kg}$ of insulin (black bars) or a similar volume of saline (white bars) in refed (RF) and control (C) groups, according to the design depicted in Fig. 1 for study design I (catch-up fat with a chow diet). Values are means $\pm \mathrm{SE} .{ }^{\circledR @ @ ~} \mathrm{P}<0.001$, effect of group $(\mathrm{C}$ vs. RF $) .{ }^{\# \# \#} \mathrm{P}<$ 0.001 , effect of treatment (insulin vs. saline). ${ }^{\times} P<0.05$; ${ }^{\times \times} P<0.001$.

tibialis anterior and soleus muscles of refed animals than in muscles of controls by 30 and $50 \%$, respectively $(P<0.05)$.

\section{AMPK phosphorylation}

The data on AMPK signaling in skeletal muscles in response to saline (basal levels) or to in vivo administration of leptin in refed and control groups, presented in Fig. 3, indicate no between-group differences in the phosphorylation of AMPK. More specifically, in vivo leptin administration did not result in significant increases in AMPK phosphorylation in tibialis anterior muscle from refed or control groups. Although in soleus muscle, the administration of leptin resulted in significant increases in AMPK phosphorylation by 1.6fold $(P<0.01)$, this response is comparable across the refed and control groups. The data on phosphorylation of ACC (a downstream target of AMPK) were similar to those obtained for AMPK phosphorylation and are presented in Supplemental Fig. 2, i.e., no change in the tibialis anterior muscle in either refed or fed control rats and comparable increases in response to leptin in soleus muscle of refed and fed animals.
Study II: catch-up fat with high-fat diet

\section{PI3K activity}

The results for the impact of increased fat in the diet on IRS-1-associated PI3K activity in tibialis anterior muscle after feeding and refeeding with the semisynthetic low-fat or high-fat (lard) diets are presented in Fig. 4, top panel. Between-diet comparisons in the fed controls (control high-fat $v s$. control low-fat) indicate no differences in basal or insulin-stimulated PI3K activity (Fig. 4, left-hand panel). In contrast, in the refed groups consuming isocaloric amounts of these two diets for the same time period of 10 days (Fig. 4, right-hand panel), there was an overall significant effect of diet on PI3K activity in tibialis anterior (refed high-fat diet vs. refed low-fat diet; $P<0.05$ by ANOVA), with a more pronounced

\section{Tibialis anterior}
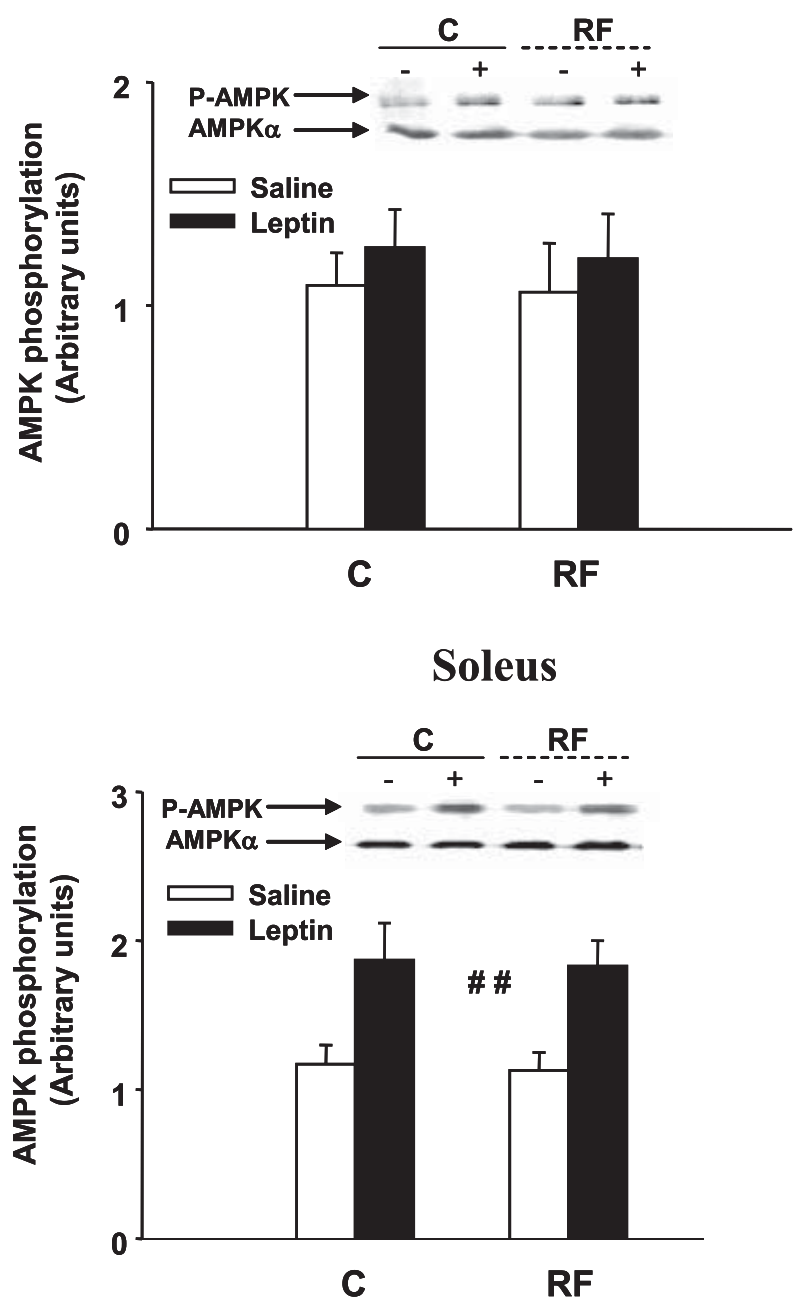

Figure 3. AMPK phosphorylation in tibialis anterior and soleus muscles in response to either an acute intravenous bolus administration of $1 \mathrm{mg} / \mathrm{kg}$ leptin (black bars) or a similar volume of saline (white bars) in refed (RF) and control (C) groups, according to the design depicted in Fig. 1 for study design I (catch-up fat with a chow diet). Values are means \pm SE. ${ }^{\# \#} P<0.01$, effect of treatment (leptin $v s$. saline). 
Tibialis anterior

Figure 4. IRS1-associated PI3K activity in tibialis anterior and soleus muscles in response to either an acute bolus intravenous administration of $10 \mathrm{U} / \mathrm{kg}$ of insulin (black bars) or a similar volume of saline (white bars) in refed (RF) or control (C) groups fed either the low-fat (LF) or high-fat (HF) diet, according to the design depicted in Fig. 1 for study design II (catch-up fat with the high-fat diet). Values are means \pm $\mathrm{SE} .{ }^{\circledR} P<0.05$, effect of diet (LF vs. HF). ${ }^{\# \#} P<$ $0.01 ;{ }^{\# \# \#} P<0.001$, effect of treatment (insulin vs. saline) by ANOVA.
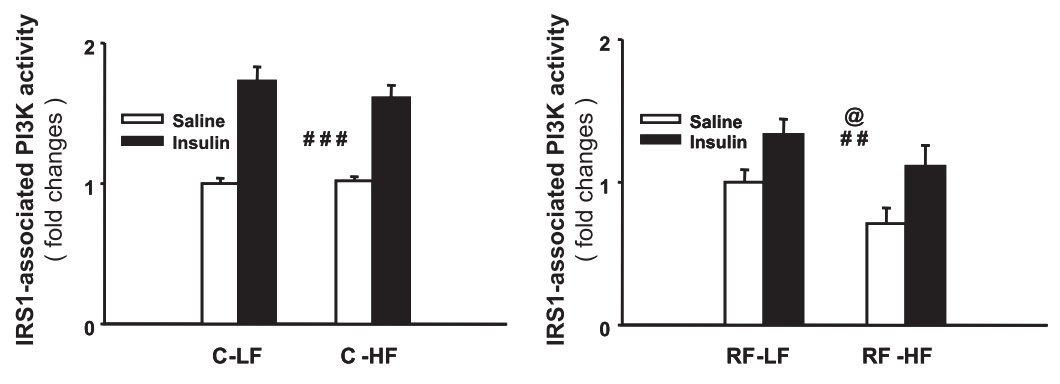

effect of the high-fat diet in lowering basal PI3K activity across saline treatment $(-30 \%, P<0.05)$ than in lowering insulin-stimulated PI3K activity $(-17 \%$, not significant). In contrast to these between-diet differences in the tibialis anterior of refed animals, no differences were observed in basal or insulin-stimulated PI3K activity in soleus muscle from refed groups nor from fed control groups, as shown in Fig. 4, bottom panel.

\section{Intramyocellular lipids and $\Delta 9$ desaturation}

After finding that skeletal muscle PI3K activity is lower during high-fat refeeding in only the tibialis anterior, in further studies we examined whether the lower PI3K activity in this muscle could be related to an elevated intramyocellular lipid content or to an up-regulation of SCD1 because both have been implicated in diminished PI3K signaling in skeletal muscle $(25,26)$ and also because of recent findings implicating a role for muscle SCD1 up-regulation in the mechanisms by which thermogenesis might be suppressed during catch-up fat with a low-fat diet (24). The data presented in Table 1, however, indicate that, like plasma free fatty acids (FFAs), the intramyocellular lipid content is not significantly different in muscles of animals consuming the high-fat diet $v s$. the low-fat diet after 10 days of isocaloric refeeding (refed high-fat diet vs. refed low-fat diet). Furthermore, SCD1 mRNA levels were not higher in response to highfat $v$ s. low-fat refeeding but, surprisingly, were markedly reduced in the refed animals consuming the high-fat diet (Table 1). These lower muscle SCD1 expressions during high-fat refeeding are associated with reductions in the SCD1-related fatty acid $\Delta 9$ desaturation index (assessed as changes in C16:1/C16:0 or in C18:1/C18:0) in both the muscle phospholipid fraction as well as in the muscle triglyceride lipid fraction (Fig. 5); similar reductions in SCD1 gene expression and the SCD1-related fatty acid $\Delta 9$ desaturation index were also observed in control animals fed with the high-fat diet.

\section{AMPK phosphorylation}

The results of AMPK signaling in skeletal muscle after feeding and refeeding with the low-fat or high-fat diets

TABLE 1. Circulating FFAs, intramyocellular lipids, and SCD1 gene expression in tibialis anterior muscle of control and refed animals

\begin{tabular}{|c|c|c|c|c|c|c|}
\hline & \multicolumn{3}{|c|}{ Fed controls } & \multicolumn{3}{|c|}{ Refed } \\
\hline & C-LF & C-HF & $t$ test & RF-LF & RF-HF & $t$ test \\
\hline Plasma FFA (mM) & $0.45 \pm 0.05$ & $0.50 \pm 0.05$ & NS & $0.48 \pm 0.05$ & $0.51 \pm 0.06$ & NS \\
\hline Intramyocellular lipids (arbitrary units) & $3.0 \pm 0.6$ & $3.6 \pm 0.7$ & NS & $1.6 \pm 0.3$ & $2.0 \pm 0.3$ & NS \\
\hline SCD1 mRNA (normalized to cyclophilin) & $7.70 \pm 1.48$ & $1.15 \pm 0.24$ & $* *$ & $21.0 \pm 3.8$ & $4.23 \pm 0.8$ & $* *$ \\
\hline
\end{tabular}

Data are mean \pm SE; C-LF and C-HF = control groups fed the low-fat or high-fat diet, respectively; RF-LF and RF-HF = refed groups consuming the low-fat or high-fat diet, respectively. Within controls or refed groups, statistical differences between groups consuming the low-fat or high-fat diets are indicated as follows: $* * P<0.01 ; \mathrm{NS}=$ not significant. 

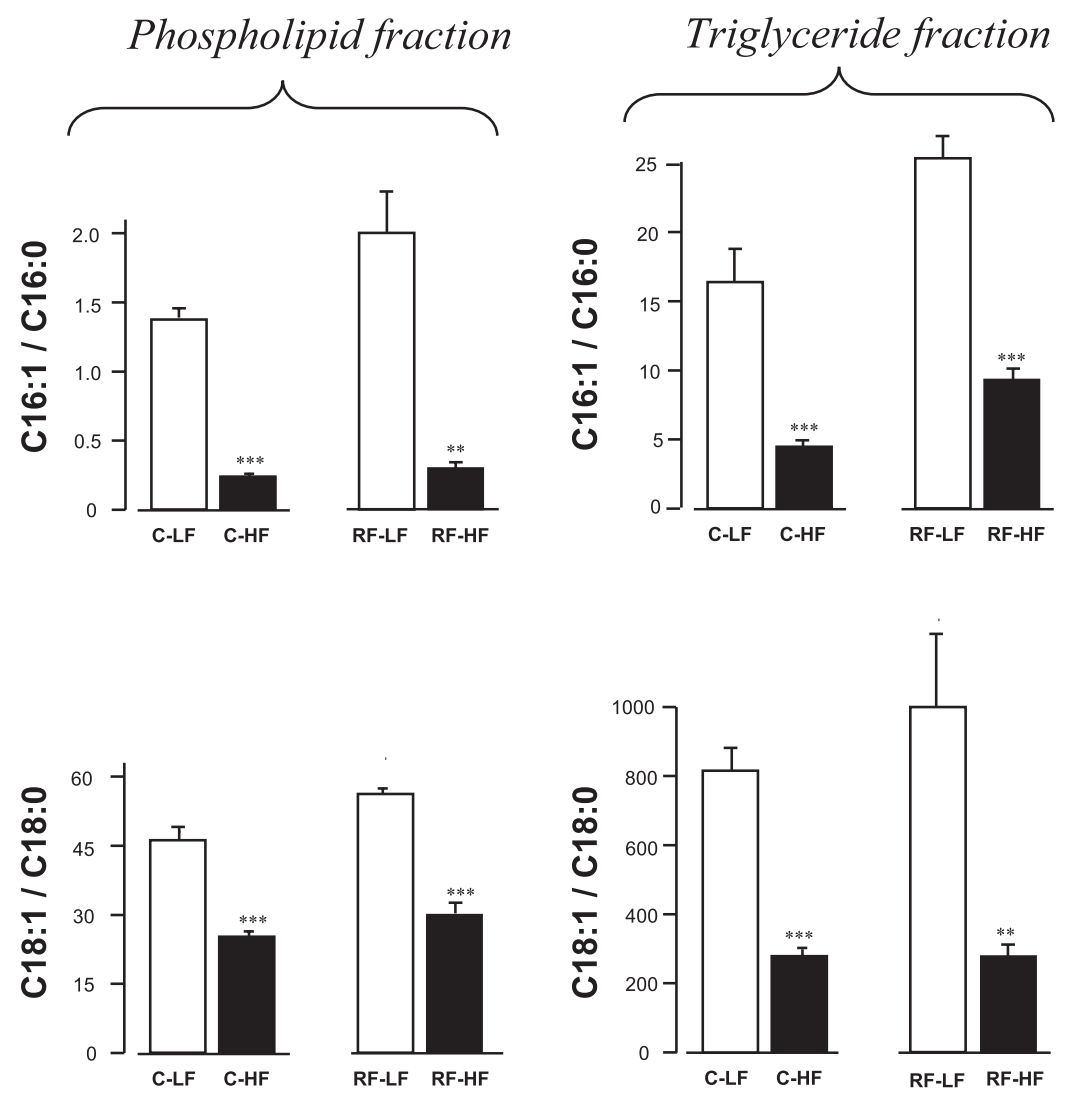

Figure 5. SCD1-related fatty acid $\Delta 9$ desaturation index (C16:1/16:0 and C18:1/C18:0) in two lipid fractions (phospholipid and triglyceride) of tibialis anterior muscle harvested from refed animals or from fed controls consuming either the low-fat or high-fat diet for 10 days according to design depicted in Fig. 1 for study design II (catch-up fat with the high-fat diet). C-LF and C-HF are control groups fed with the low-fat or high-fat diet, respectively; RF-LF and RF-HF are refed groups consuming the low-fat or high-fat diet, respectively. Values are means $\pm \mathrm{SE} . * * P<0.01$; $* * * P<0.001$, betweendiet differences.

for 10 days are presented in Fig. 6 for both tibialis anterior and soleus. Comparisons across the fed controls (control high-fat diet $v s$. control low-fat diet) indicated no between-diet differences in basal or leptin-

stimulated AMPK in both tibialis anterior and soleus. In contrast, comparisons across the refed groups (refed high-fat diet $v s$. refed low-fat diet) indicate an effect of the high-fat diet in lowering AMPK phosphorylation in

\section{Tibialis anterior}
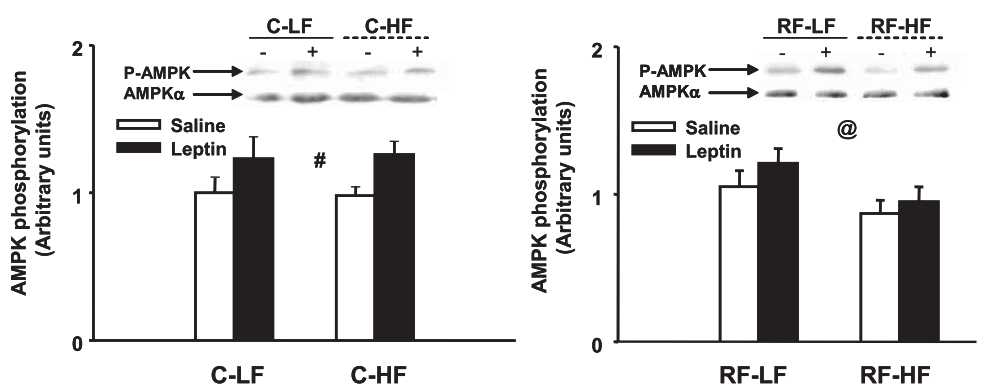

Figure 6. AMPK phosphorylation in tibialis anterior and soleus muscles in response to either an acute intravenous bolus administration of $1 \mathrm{mg} / \mathrm{kg}$ leptin (black bars) or a similar volume of saline (white bars) in refed $(\mathrm{RF})$ or control $(\mathrm{C})$ groups fed either the low-fat (LF) or high-fat (HF) diet, according to design depicted in Fig. 1 for study II (catch-up fat with the high-fat diet). Values are means $\pm \mathrm{sE} .{ }^{\circledR} P<$

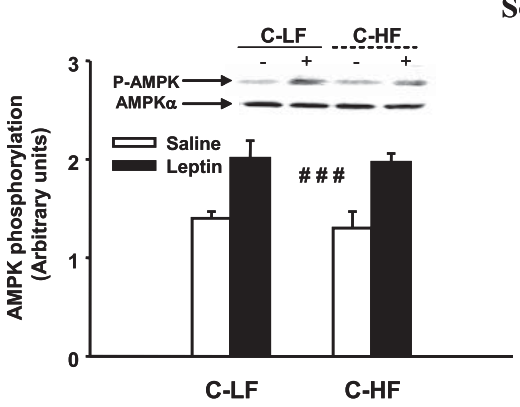

Soleus

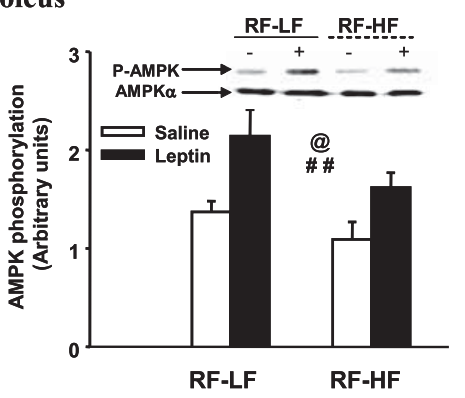
${ }^{\# \# \# P} P 0.001$, effect of treatment (leptin $v s$. saline). 
both tibialis anterior (by 15-20\%) and in soleus (by 20-25\%), with ANOVA indicating an overall significant difference $(P<0.05)$ across diets and with the reductions in basal AMPK accounting for $>50 \%$ of the reductions in leptin-stimulated AMPK phosphorylation. Similarly, the phosphorylation of ACC was also found to be lower, specifically in animals refed the high-fat diet than in those fed the low-fat diet in both muscles (Supplemental Fig. 3), with the between-diet differences in ACC phosphorylation, as for AMPK phosphorylation, being observed only in muscles from refed animals but not in those from fed controls.

\section{Thermogenic hormones}

To test whether our findings of impaired muscle AMPK during refeeding with the high-fat diet might be associated with lower circulating levels of thermogenic hormones that have been implicated in AMPK activation in skeletal muscle, namely leptin (31), the thyroid hormone T3 (36), and catecholamines $(31,37)$, we also determined plasma concentrations of leptin and T3, as well as urinary excretion rates of catecholamines for indices of altered sympathetic and adrenal medullary activity. As shown in Table 2, none of these hormones were found to be lower during high-fat refeeding. On the contrary, plasma leptin concentrations and urinary norepinephrine excretion rates were significantly higher during high-fat refeeding than during low-fat refeeding (refed high-fat diet vs. refed low-fat diet) by $55 \% \quad(P<0.001)$ and by $17 \%(P<0.01)$, respectively; these effects of the high-fat diet in increasing circulating leptin concentration and urinary norepinephrine excretion rate being observed only in the refed group and not in fed controls (Table 2).

\section{DISCUSSION}

Using a rat model of semistarvation-refeeding in which catch-up fat is driven only by suppressed thermogenesis, we demonstrate here that basal and leptin-induced AMPK signaling and basal or insulin-stimulated IRS1associated PI3K activity are reduced during catch-up fat and precede a detectable elevation in circulating FFAs or in intramyocellular lipid content. These findings support the hypothesis that diminished skeletal muscle PI3K and AMPK signaling may indeed be of central importance in the suppression of thermogenesis that favors the rapid rebuilding of the fat stores after caloric restriction.

\section{Catch-up fat with low-fat diet}

A comparison between refed and fed control animals consuming isocaloric amounts of a low-fat (chow) diet indicates no detectable differences in AMPK phosphorylation but consistently lower IRS1-associated PI3K activity in skeletal muscle during early catch-up fat. PI3K activity is significantly lower during refeeding both in the fast-twitch glycolytic muscle (tibialis anterior) and in the slow-twitch oxidative muscle (soleus). Furthermore, lower muscle PI3K activity in refed than in control animals is observed in the unstimulated (basal) state as well as after in vivo insulin administration. Taken together, these data demonstrate that during catch-up fat, skeletal muscle has a reduced pool of IRS1-associated PI3K activity and a defective induction by insulin. These findings, together with our previous studies of ex vivo microcalorimetry implicating a role for PI3K activity and insulin in skeletal muscle thermogenesis (27-29), suggest that the defective IRS1-associated PI3K activity in both basal and in response to insulin stimulation might be important in the suppressed thermogenesis that favors catch-up fat. Moreover, the lower insulin-stimulated PI3K activity in skeletal muscle during refeeding suggests that a defect in the early steps of insulin signaling might be the molecular defect linking suppressed thermogenesis and insulin resistance during catch-up fat. This hypothesis is supported by studies with a euglycemic-hyperinsulinemic clamp showing that insulin-stimulated glucose utilization in skeletal muscle is lower in refed animals than in controls, a reduction that precedes excess body fat, elevated intramyocellular lipids, or elevated circulating FFAs (11-12), thereby suggesting that insulin resistance in skeletal muscle during catch-up fat occurs before overt lipotoxic stress.

TABLE 2. Basal (postabsorptive) level of plasma hormones and 24-h urinary excretion of catecholamines in control and refed animals

\begin{tabular}{|c|c|c|c|c|c|c|}
\hline & \multicolumn{3}{|c|}{ Fed controls } & \multicolumn{3}{|c|}{ Refed } \\
\hline & C-LF & C-HF & $t$ test & RF-LF & RF-HF & $t$ test \\
\hline \multicolumn{7}{|l|}{ Plasma hormones } \\
\hline Leptin $(\mathrm{ng} / \mathrm{ml})$ & $1.92 \pm 0.33$ & $2.59 \pm 0.37$ & NS & $2.58 \pm 0.29$ & $4.00 \pm 0.24$ & $* *$ \\
\hline $\mathrm{T} 3(\mathrm{pg} / \mathrm{ml})$ & $3.37 \pm 0.18$ & $2.92 \pm 0.09$ & NS & $2.71 \pm 0.08$ & $3.02 \pm 0.22$ & NS \\
\hline \multicolumn{7}{|l|}{ Urinary catecholamines } \\
\hline Norepinephrine (nmol/day) & $6.62 \pm 0.18$ & $5.61 \pm 0.72$ & NS & $4.78 \pm 0.12$ & $5.58 \pm 0.15$ & $* *$ \\
\hline Epinephrine (nmol/day) & $1.34 \pm 0.12$ & $0.61 \pm 0.06$ & $* * *$ & $0.51 \pm 0.02$ & $0.49 \pm 0.02$ & NS \\
\hline
\end{tabular}

Data are mean \pm SE. C-LF and C-HF = control groups fed the low-fat or high-fat diet, respectively; RF-LF and RF-HF $=$ refed groups consuming the low-fat or high-fat diet, respectively. Within controls or refed groups, statistical differences between groups consuming the low-fat or high-fat diets are indicated as follows: $* * P<0.01 ; * * * P<0.001$. NS $=$ not significant. 


\section{Catch-up fat with high-fat diet}

Further support of a role for impaired PI3K activity in the suppression of thermogenesis driving catch-up fat can also be derived from our findings in animals refed with a high-fat diet. We have shown previously (11) and confirmed here (Fig. 1) that isocaloric consumption of this diet rich in saturated animal fat (compared with a diet low in fat) results in a more pronounced reduction in energy expenditure in refed animals than in fed controls, thereby revealing an exacerbated suppression of thermogenesis induced by dietary fat during refeeding. Using the same experimental design, we show here that basal and insulin-stimulated IRS1-associated PI3K activity in tibialis anterior is also diminished in response to increased dietary fat, specifically during refeeding. This reduction in PI3K activity in tibialis anterior, a fast-twitch muscle whose fiber composition reflects that observed for the bulk of skeletal mass in the rat (38), can also be dissociated in this study from an elevation in circulating FFAs, in intramyocellular lipid content, or in SCD1 up-regulation, all of which are generally associated with obesity-induced insulin resistance and lipotoxicity (39). These findings further suggest that suppressed thermogenesis and skeletal muscle insulin sensitivity, both of which require IRS1-associated PI3K activity, are interdependent during catch-up fat.

A role for impaired AMPK signaling in skeletal muscle can also be suggested in the exacerbated suppression of thermogenesis induced by a high-fat diet during refeeding. Our previous studies in intact muscles ex vivo showed that AMPK is required for the direct stimulatory effects of both leptin and corticotropinreleasing hormone on skeletal muscle thermogenesis $(28,29)$. Furthermore, 5-aminoimidazole-4-carboxamide riboside, a potent AMPK inducer, can also stimulate skeletal muscle thermogenesis (28). In this study, we show that AMPK phosphorylation, both basal (unstimulated) and in response to in vivo leptin administration, is lower in both tibialis anterior and soleus muscle of animals refed with a high-fat diet compared with those refed with a low-fat diet. Such impairments in AMPK cannot be consequential to low circulating levels of hormones implicated in the control of AMPK signaling in skeletal muscle-leptin (31), thyroid hormone T3 (36), and catecholamines (37) - because plasma concentrations of these hormones are found to be similar or higher during high-fat refeeding than during low-fat refeeding. The observation that AMPK activation in skeletal muscle is decreased in rats refed with a high-fat diet but not with a low-fat diet is consistent with previous reports of defective AMPK signaling in obese and insulin-resistant Zucker rats $(40,41)$ and in rats made obese by feeding with a high-fat diet for several months (42). In our study, however, the defective AMPK phosphorylation in response to dietary fat is observed here specifically in refed animals and not in fed controls (Fig. 6) and before a detectable elevation in circulating FFA and intramyocellular lipid content. These data therefore strongly suggest that the impair- ment of AMPK activation during high-fat refeeding is an early event that might contribute to the exaggerated suppression of thermogenesis favoring catch-up fat and that refeeding predisposes to the deleterious effects of dietary fat on skeletal muscle AMPK signaling.

\section{Dissociation between muscle SCD1 and thermogenesis}

A role for skeletal muscle SCD1 in adaptive thermogenesis was previously proposed on the basis that increased SCD1 gene expression and an increased fatty acid $\Delta 9$ desaturation index (an index for SCD1 activity) correlated with suppressed thermogenesis during refeeding with the standard chow diet (low in fat and high in carbohydrates) (24). We show here for the first time that both SCD1 gene expression and the fatty acid $\Delta 9$ desaturation index in skeletal muscle are markedly down-regulated by a high-fat diet, despite the fact that energy expenditure is lower with the high-fat than the low-fat diet in both refed and fed groups (Fig. 1). It is possible that such repression of muscle SCD1 by the high-fat diet plays a protective role against lipotoxicity in skeletal muscle, a view that is consistent with our data here showing no significant increase in intramyocellular lipids and also with the observation that SCD1 knockout mice display lower muscle lipid content than wild-type controls (26). Nevertheless, our data dissociate skeletal muscle SCD1 activity from the control of whole-body thermogenesis during high-fat consumption. Thus, although knockout of the gene expressing SCD1 results in an hypermetabolic phenotype, and that up-regulation of SCD1 in skeletal muscle correlates with suppressed thermogenesis favoring catch-up fat with a low-fat diet, down-regulation of muscle SCD1 in rats refed (or fed) with a high-fat diet does not increase whole-body thermogenesis. Taken together, these data demonstrate that muscle SCD1 up-regulation per se is not a general requirement for the suppression of thermogenesis that causes catch-up fat.

The main conclusion of the present studies is that IRS1-associated PI3K activity and AMPK activation are defective in skeletal muscle of rat during the early phase of catch-up fat driven by suppressed thermogenesis. Although the exact sequence of events between suppressed thermogenesis and reduced PI3K and AMPK activities remains to be clarified, our view is that suppressed thermogenesis and defective PI3K and AMPK signaling are likely to be interdependent and that these pathways play a major role in the control of skeletal muscle thermogenesis. This hypothesis is consistent with the observations that knockout of the $S c d 1$ gene or at the 56 kinase-1 gene locus in mice leads to resistance to obesity through an elevation in wholebody thermogenesis with enhanced IRS1-associated PI3K activity and AMPK activation in skeletal muscle $(22,23,43,44)$. 


\section{A molecular mechanism for thrifty metabolism driving catch-up fat}

Which molecular effectors of skeletal muscle thermogenesis might be under the control of PI3K and AMPK signaling and which factors contribute to early impairments in these signaling pathways during catch-up fat are questions that need to be addressed in the future. Of particular interest is the recent demonstration that rats showing catch-up fat due to suppressed thermogenesis also show diminished mitochondrial mass and oxidative capacity specifically in the subsarcolemmal compartment of skeletal muscle (45). Given the important role that subsarcolemmal mitochondria have for bioenergetic support of signal transduction and substrate transport (46), the possibility arises that a deficiency in this subpopulation of mitochondria could contribute to impaired PI3K and AMPK signaling. Although a reduction in the number of subsarcolemmal mitochondria per se is likely to contribute to the suppression of thermogenesis that favors catch-up fat, impaired PI3K and AMPK signaling could also slow down a futile cycle between de novo lipogenesis and mitochondrial lipid oxidation $(28,29)$.

According to the model presented in Fig. 7A, PI3K is required for efficient flux of glucose through de novo lipogenesis, and AMPK is required for a sustained flux of de novo synthesized fatty acids through mitochondrial $\beta$-oxidation (47). This model can be used to explain the mechanisms by which thermogenesis is suppressed in skeletal muscle during catch-up fat. The impact of defective AMPK and PI3K observed during catch-up fat on this futile cycle is illustrated in Fig. $7 A, B$ for refeeding with a low-fat diet and Fig. $7 C, D$ for refeed- ing with the high-fat diet. Thus, during catch-up fat with a low-fat diet (Fig. 7A, B), the reduction in PI3K will have an impact on several key control points. Being crucial for glucose uptake, impairments in PI3K signaling will indirectly interfere with substrate cycling possibly by decreasing the levels of NADPH, which is required for lipogenesis and by decreasing anaplerosis of the Krebs' cycle. Furthermore, PI3K can also exert control over fatty acid synthase $(48,49)$, and therefore a reduction in PI3K activity might reduce the flux of substrates through de novo lipogenesis leading to a slowdown of the substrate cycle. This model also takes into account our earlier finding that SCD1 is upregulated in skeletal muscle of rats showing catch-up fat during refeeding with a low-fat (high-carbohydrate) diet (24). Such an elevated skeletal muscle SCD1, by desaturating the products of de novo lipogenesis and diverting them away from mitochondrial $\beta$-oxidation, could also contribute to decreased substrate cycling between de novo lipogenesis and lipid oxidation; hence, the suppression of thermogenesis that promotes catch-up fat (Fig. 7A, B). However, during catch-up fat with a high-fat diet (Fig. $7 C, D$ ), SCD1 up-regulation is blunted, but the reductions in IRS1-associated PI3K signaling, together with defective AMPK activation, will inhibit the substrate flux through both lipogenic and $\beta$-oxidation pathways that constitute the substrate cycle between de novo lipogenesis and lipid oxidation, thereby explaining the exacerbated suppression of thermogenesis and excess body fat observed during high-fat refeeding.

The model presented in Fig. 7 depicts a critical role for diminished muscle PI3K and AMPK signaling in the molecular mechanisms leading to suppressed thermo-
Figure 7. Model illustrating that in the fed state, repeated recycling of acetyl-CoA through the flux of substrates across de novo lipogenesis followed by mitochondrial $\beta$-oxidation constitutes an energy dissipating "substrate cycling" in skeletal muscle $(28,29,47)$, with PI3K, AMPK, and SCD1 as control points in this effector of thermogenesis $(A)$. During refeeding with the low-fat diet $(A, B)$, diminished PI3K activity (by diminishing glucose entry and glucose flux through de novo lipogenesis), together with an elevated SCD1 (24) (by enhancing the desaturation of de novo synthesized fatty acids and hence diverting them away from pathways of mitochondrial $\beta$-oxidation) would effectively slow down this thermogenic substrate cycling mechanism, thereby leading to suppressed thermogenesis (energy conservation) that drives catch-up fat. During refeeding with the high-fat diet $(C, D)$, the elevation in SCD1 is blunted, but the exacerbated reduction in PI3K activity, together with diminished AMPK activity, will lead to inhibition of substrate flux through both lipogenic and $\beta$-oxidation pathways that constitute the substrate cycling between de novo lipogenesis and lipid oxidation, thereby contributing to an exacerbated suppression of thermogenesis and excess fat deposition. Act-CoA = acetyl CoA; LCSFA = long-chain saturated fatty acids; MUFA $=$ monounsaturated fatty acids.
Low Fat Diet
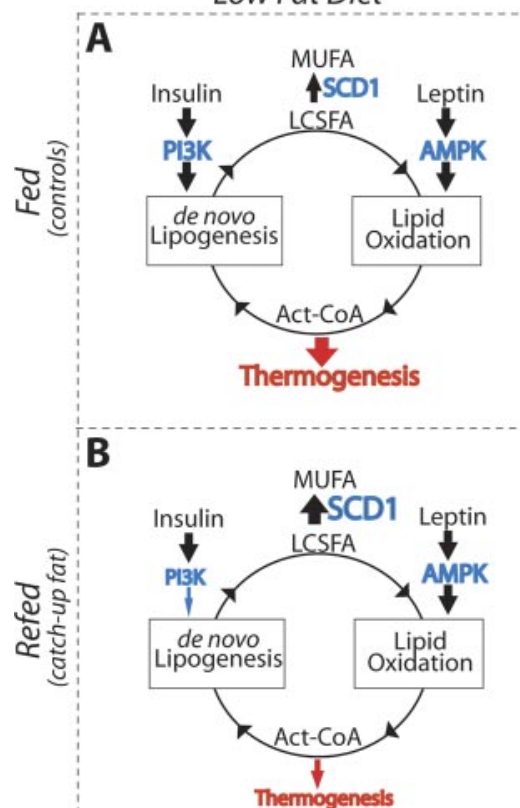

High Fat Diet
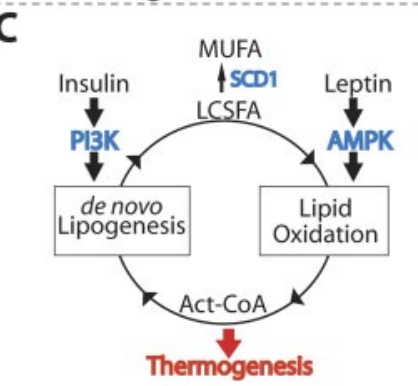

D

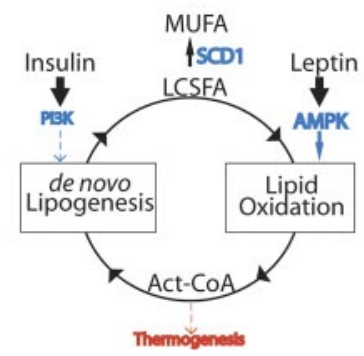


genesis, which accelerates fat recovery and furthermore sensitizes muscle to dietary fat-induced impairments in PI3K and AMPK signaling. It follows that impairments in these signaling pathways may be part of the molecular mechanisms by which the thrifty metabolism that accelerates fat recovery after caloric restriction confers enhanced susceptibility to obesity and insulin resistance and hence promotes the pathogenesis of metabolic syndrome in individuals who had experienced frequent weight cycling or catch-up growth earlier in life.

We thank Aldo Tempini and Marie-Françoise Baeriswyl for excellent technical assistance. This project was funded by the Swiss National Science Research Foundation (grants 3200-B0102156 and 3200B0-113634), the Roche Research Foundation, and the Swiss Heart Foundation.

\section{REFERENGES}

1. Keys, A., Brozek, J., Henschel, A., Mickelson, O., and Taylor, H. L. (1950) The Biology of Human Starvation, University of Minnesota Press, Minneapolis

2. Dulloo, A. G., and Jacquet, J. (1998) Adaptive reduction in basal metabolic rate in response to food deprivation in human: a role for feedback signal from the fat stores. Am. J. Clin. Nutr. 68, $599-606$

3. Weyer, C., Walford, R. L., Harper, I. T., Milner, M., MacCallum, T., Tataranni, P. A., and Ravussin, E. (2000) Energy metabolism after $2 \mathrm{y}$ of energy restriction: the Biosphere 2 experiment. Am. J. Clin. Nutr. 72, 946-953

4. Boyle, P. C., Storlien, L. H., and Keesey, R. E. (1978) Increased efficiency of food utilization following weight loss. Physiol. Behav. 21, 261-264

5. Hill, J. O., Fried, S. K., and Digirolamo, M. (1984) Effects of fasting and restricted refeeding on utilization of ingested energy in rats. Am. J. Physiol. 247, R318-R327

6. Harris, R. B. S., and Martin, R. J. (1984) Recovery of body weight from below 'Set Point' in mature female rats. J. Nutr. 114, $1143-1150$

7. MacLean, P. S., Higgins, J. A., Johnson, G. C., Fleming-Elder, B. K., Donahoo, W. T., Melanson, E. L., and Hill, J. O. (2004) Enhanced metabolic efficiency contributes to weight regain after weight loss in obesity-prone rats. Am. J. Physiol. 287, R1306-R1315

8. Evans, S. A., Messina, M. M., Knight, W. D., Parsons, A. D., and Overton, J. M. (2005) Long-Evans and Sprague-Dawley rats exhibit divergent responses to refeeding after caloric restriction. Am. J. Physiol. 288, R1468-R1476

9. Dulloo, A. G., and Girardier, L. (1990) Adaptive changes in energy expenditure during refeeding following low calorie intake: evidence for a specific metabolic component favouring fat storage. Am. J. Clin. Nutr. 52, 415-420

10. Dulloo, A. G., and Jacquet, J. (2001) An adipose-specific control of thermogenesis in body weight regulation. Int. J. Obes. Relat. Metab. Disord. 25(Suppl. 5), S22-S29

11. Crescenzo, R., Samec, S., Antic, V., Rohner-Jeanrenaud, F., Seydoux, J., Montani, J. P., and Dulloo, A. G. (2003) A role for suppressed thermogenesis favouring catch-up fat in the pathophysiology of catch-up growth. Diabetes 52, 1090-1097

12. Cettour-Rose, P., Samec, S., Russell, A. P., Summermatter, S., Mainieri, D., Carrillo-Theander, C., Montani, J. P., Seydoux, J., Rohner-Jeanrenaud, F., and Dulloo, A. G. (2005) Redistribution of glucose from skeletal muscle to adipose tissue during catch-up fat: A link between catch-up growth and later metabolic syndrome. Diabetes 54, 751-756

13. Dulloo, A. G., Jacquet, J., and Montani, J. P. (2002) Pathways from weight fluctuation to metabolic disease: focus on maladaptive thermogenesis during catch-up fat. Int. J. Obes Relat Metab. Disord 26 (Suppl. 2), S46-S57

14. Dulloo, A. G., Seydoux, J., and Girardier, L. (1995) Dissociation of enhanced efficiency of fat deposition during weight recovery from sympathetic control of thermogenesis. Am. J. Physiol. 269, R365-R369

15. Boss, O., Samec, S., Kuhne, F., Bijlenga, P., AssimacopoulosJeannet, F., Seydoux, J., Giacobino, J. P., and Muzzin, P. T. (1998) Uncoupling protein-3 expression in rodent skeletal muscle is modulated by food intake but not by changes in environmental temperature. J. Biol. Chem. 273, 5-8

16. Samec, S., Seydoux, J., and Dulloo, A. G. (1998) Role of UCP homologues in skeletal muscle and brown adipose tissue: mediators of thermogenesis or regulators of lipids as fuel substrate? FASEB J. 12, 715-724

17. Cadenas, S., Buckingham, J. A., Samec, S., Seydoux, J., Din, N., Dulloo, A. G., and Brand, M. D. (1999) UCP2 and UCP3 rise in starved rat skeletal muscle but mitochondrial proton conductance is unchanged. FEBS Lett. 462, 257-260

18. Harper, M. E., Dent, R.M., Bezaire, V., Antoniou, A., Gauthier, A., Monemdjou, S., and McPherson, R. (2001) UCP3 and its putative function: consistencies and controversies. Biochem. Soc. Trans. 29 (Pt 6), 768-773

19. Crescenzo, R., Mainieri, D., Solinas, G., Montani, J. P., Seydoux, J., Liverini, G., Iossa, S., and Dulloo, A. G, (2003) Skeletal muscle mitochondrial oxidative capacity and uncoupling protein 3 are differently influenced by semistarvation and refeeding. FEBS Lett. 544, 138-142

20. Dulloo, A. G., Seydoux, J. S., and Jacquet, J. (2004) Adaptive thermogenesis and uncoupling proteins: a reappraisal of their roles in fat metabolism and energy balance. Physiol. Behav. 83, 587-602

21. Cannon, B, Shabalina, I. G., Kramarova, T.V., Petrovic, N., and Nedergaard, J. (2006) Uncoupling proteins: a role in protection against reactive oxygen species-or not? Biochim. Biophys. Acta 1757, 449-458

22. Cohen, P., Miyazaki, M., Socci, N. D., Hagge-Greenberg, A., Liedtke, W., Soukas, A. A., Sharma, R., Hudgins, L. C., Ntambi, J. M., and Friedman, J. M. (2002) Role for stearoyl-CoA desaturase-1 in leptin-mediated weight loss. Science 297, 240-243

23. Ntambi, J. M., Miyazaki, M., Stoehr, J. P., Lan, H., Kendziorski, C. M., Yandell, B. S., Song, Y., Cohen, P., Friedman, J. M., and Attie, A. D. (2002) Loss of stearoyl-CoA desaturase-1 function protects mice against adiposity. Proc. Natl. Acad. Sci. U. S. A. 99 11482-11486

24. Mainieri, D., Summermatter, S., Seydoux, J., Montani, J. P., Rusconi, S., Russell, A. P., Boss, O., Buchala, A. J., and Dulloo, A. G. (2006) A role for stearoyl-CoA desaturase in control of thermogenesis. FASEB J. 20, 1751-1753

25. Rahman, S. M., Dobrzyn, A., Dobrzyn, P., Lee, S. H., Miyazaki, M., and Ntambi, J. M. (2003) Stearoyl-CoA desaturase 1 deficiency elevates insulin-signaling components and down-regulates protein-tyrosine phosphatase 1B in muscle. Proc. Natl. Acad. Sci. U. S. A. 100, 11110-11115

26. Dobrzyn, A., Dobrzyn, P., Lee, S. H., Miyazaki, M., Cohen, P., Asilmaz, E., Hardie, D. G., Friedman, J. M., and Ntambi, J. M. (2005) Stearoyl-CoA desaturase-1 deficiency reduces ceramide synthesis by downregulating serine palmitoyltransferase and increasing $\beta$-oxidation in skeletal muscle. Am. J. Physiol. 288, E599-E607

27. Dulloo, A. G., Stock, M. J., Solinas, G., Boss, O., Montani, J. P., and Seydoux, J. (2002) Leptin directly stimulates thermogenesis in skeletal muscle. FEBS Lett. 515, 109-113

28. Solinas, G., Summermatter, S., Mainieri, D., Gubler, M., Pirola, L., Wymann, M. P., Rusconi, S., Montani, J. P., Seydoux, J., and Dulloo, A. G. (2004) The direct effect of leptin on skeletal muscle thermogenesis is mediated by substrate cycling between de novo lipogenesis and lipid oxidation. FEBS Lett. 577, 539-544

29. Solinas, G., Summermatter, S., Mainieri, D., Gubler, M., Montani, J. P., Seydoux, J., Smith, S. R., and Dulloo, A. G. (2006) Corticotropin-releasing hormone directly stimulates thermogenesis in skeletal muscle possibly through substrate cycling between de novo lipogenesis and lipid oxidation. Endocrinology 147, 31-38

30. Kim, Y. B., Uotani, S., Pierroz, D. D., Flier, J. S., and Kahn, B. B. (2002) In vivo administration of leptin activates signal transduction directly in insulin-sensitive tissues: overlapping but distinct pathways from insulin. Endocrinology 141, 2328-2339

31. Minokoshi, Y., Kim, Y. B., Peroni, O. D., Fryer, L. G., Muller, C., Carling, D., and Kahn, B. B. (2002) Leptin stimulates fatty-acid 
oxidation by activating AMP-activated protein kinase. Nature 415, 339-343

32. Pirola, L., Zvelebil, M. J., Bulgarelli-Leva, G., Van Obberghen, E., Waterfield, M. D., and Wymann, M. P. (2001) Activation loop sequences confer substrate specificity to phosphoinositide 3-kinase $\alpha(\mathrm{PI} 3 \mathrm{~K} \alpha)$ : functions of lipid kinase-deficient PI3K $\alpha$ in signalling. J. Biol. Chem. 276, 21544-21554

33. Russell, A. P., Gastaldi, G., Bobbioni-Harsch, E., Arboit, P., Gobelet, C., Dériaz, O., Golay, A., Witztum, J. L., and Giacobino, J. P. (2003) Lipid peroxidation in skeletal muscle of obese as compared to endurance trained humans: a case of good vs bad lipids? FEBS Lett. 551, 104-106

34. Miller, D. S., and Payne, P. R. (1959) A ballistic bomb calorimeter. Br. J. Nutr. 13, 501-508

35. Entenman, C. General procedures for separating components of tissue. Methods Enzymol. 3, 299-317, 1957

36. Park, S. H., Paulsen, S. R., Gammon, S. R., Mustard, K. J., Hardie, D. G., and Winder, W. W. (2002) Effects of thyroid state on AMP-activated protein kinase and acetyl-CoA carboxylase expression in muscle. J. Appl. Physiol. 93, 2081-2088

37. Hutchinson, D. S., and Bengtsson, T. (2006) AMP-activated protein kinase activation by adrenoceptors in L6 skeletal muscle cells: mediation by $\alpha_{1}$-adrenoceptors causing glucose uptake. Diabetes 55, 682-690

38. Ariano, M. A., Armstrong, R. B., and Edgerton, V. R. (1973) Hindlim muscle fiber populations of five mammals. J. Histochem. Cytochem. 21, 51-55

39. Slawick, M., and Vidal-Puig, A. J. (2006) Lipotoxicity, overnutrition and energy metabolism in aging. Ageing Res. Rev. 5, 144-164

40. Barnes, B. R., Ryder, J. W., Steiler, T. L., Fryer, L. G., Carling, D., and Zierath, J. R. (2002) Isoform-specific regulation of $5^{\prime}$ AMP-activated protein kinase in skeletal muscle from obese Zucker $(f a / f a)$ rats in response to contraction. Diabetes 51, 2703-2708

41. Sriwijitkamol, A., Ivy, J. L., Christ-Roberts, C., DeFronzo, R. A., Mandarino, L. J., and Musi, N. (2006) LKB1-AMPK signaling in muscle from obese insulin-resistant Zucker rats and effects of training. Am. J. Physiol. 290, E925-E932
42. Liu, Y., Wan, Q., Guan, Q., Gao, L., and Zhao, J. (2006) High-fat diet feeding impairs both the expression and activity of AMPKa in rats' skeletal muscle. Biochem. Biophys. Res. Commun. 339, $701-707$

43. Um, S. H., Frigerio, F., Watanabe, M., Picard, F., Joaquin, M. Sticker, M., Fumagalli, S., Allegrini, P. R., Kozma, S. C., Auwerx, J., and Thomas, G. (2004) Absence of S6K1 protects against ageand diet-induced obesity while enhancing insulin sensitivity. Nature 431, 200-205

44. Aguilar, V., Alliouachene, S., Sotiropoulos, A., Sobering, A., Athea, Y., Djouadi, F., Miraux, S., Thiaudière, E., Foretz, M., Viollet, B., Diolez, P., Bastin, J., Benit, P., Rustin, P., Carling, D., Sandri, M., Ventura-Clapier, R., and Pende, M. (2007) S6 kinase deletion suppresses muscle growth adaptations to nutrient availability by activating AMP kinase. Cell Metab. 5, 476-487

45. Crescenzo, R., Lionetti, L., Mollica, M. P., Ferraro, M., D’Andrea, E., Mainieri, D., Dulloo, A. G., Liverini, G., and Iossa, S. (2006) Altered skeletal muscle subsarcolemmal mitochondrial compartment during catch-up fat after caloric restriction. Diabetes 55, 2286-2293

46. Hood, D. (2001) Plasticity in skeletal, cardiac, and smooth muscle: contractile activity-induced mitochondrial biogenesis in skeletal muscle. J. Appl. Physiol. 90, 1137-1157

47. Dulloo A. G., Gubler, M., Montani, J. P, Seydoux, J., and Solinas, G. (2004) Substrate cycling between de novo lipogenesis and lipid oxidation: a thermogenic mechanism against skeletal muscle lipotoxicity and glucolipotoxicity. Int. J. Obes. 28(Supp 4), S29-S37

48. Wang, D., and Sul, H. S. (1998) Insulin stimulation of the fatty acid synthase promoter is mediated by the phosphatidylinositol 3-kinase pathway: involvement of protein kinase B/Akt. J. Biol. Chem. 273, 25420-25426

49. Yang, Y. A., Morin, P. J., Han, W. F., Chen, T., Bornman, D. M., Gabrielson, E. W., and Pizer, E. S. (2003) Regulation of fatty acid synthase expression in breast cancer by sterol regulatory element binding protein-1c. Exp. Cell. Res. 282, 132-137 